Check for updates

Cite this: RSC Adv., 2017, 7, 24841

Received 28th February 2017

Accepted 3rd May 2017

DOI: $10.1039 / c 7 r a 02495 a$

rsc.li/rsc-advances

\title{
Unexpected aggregation induced circular dichroism, circular polarized luminescence and helical assembly from achiral hexaphenylsilole (HPS) $\uparrow$
}

\author{
Shan Xue, ${ }^{a}$ Luming Meng, ${ }^{\mathrm{b}}$ Rongsen Wen, ${ }^{a}$ Lin Shi, ${ }^{\mathrm{c}}$ Jacky W. Lam, ${ }^{\mathrm{d}}$ Zhiyong Tang, ${ }^{\mathrm{C}}{ }^{\mathrm{c}}$ \\ Bing Shi Li (D) and Ben Zhong Tang*d
}

\begin{abstract}
This work provides the finding of aggregation-induced circular dichroism (AICD), optical properties and helical assemblies of $\pi-\pi$ conjugated molecule hexaphenylsilole (HPS). As an achiral molecule, HPS was found to exhibit unusual AICD and circular polarized luminescence (CPL). Upon aggregation, it selfassembled into fluorescent helical nanofiber and nanotubes. Theoretical modeling suggests that van der Waals interaction between HPS monomers plays an essential role in the formation of helical nanofibers. These kinds of molecules have important applications given the increasing demand of miniaturized optics and electronic devices.
\end{abstract}

\section{Introduction}

Helical assemblies are the critical level of the hierarchical architectures of molecules, which bridge the elementary building blocks and macromolecular properties of molecules. ${ }^{1}$ Helical assemblies play a pivotal role in determining the optical properties of the molecules. To form helical assemblies, chiral centers are usually necessary in the chemical structure of the molecules to induce an asymmetric arrangement of the molecules, which is further amplified on the higher level of architectures as supramolecular helical assemblies. ${ }^{2}$ Besides the chiral center induced helical assemblies, mixing achiral molecules with chiral additives, solvent or metal ions can also help to induce the formation of helical assemblies. ${ }^{3}$ The addition of chiral additives may also guide the achiral molecules to form helical assemblies by obeying a sergeant-solider rule; while metal ions induce achiral amphiphilic molecules to give helical assemblies by forming coordination complexes. External

${ }^{a}$ Key Laboratory of New Lithium-Ion Battery and Mesoporous Materials, Department of Chemistry and Environmental Engineering, Shenzhen University, 3688 Nanhai Road, Shenzhen 518060, China. E-mail: phbingsl@szu.edu.cn; Fax: +86-755-26536141; Tel: +86-755-26558094

${ }^{b}$ Beijing National Laboratory for Molecular Sciences, College of Chemistry and Molecular Engineering, Beijing University, No. 5 Yiheyuan Road Haidian District, Beijing, 100871 Beijing, China

'National Center for Nanoscience and Technology (NCNST), No. 11 Zhong Guan Cun Bei Yi Tiao, 100190, Beijing, China

${ }^{d}$ Hong Kong University of Science \& Technology (HKUST), Shenzhen Research Institute, No. 9 Yuexing 1st RD, South Area, Hi-tech Park, Nanshan, Shenzhen 518057, China. E-mail: tangbenz@ust.hk

$\dagger$ Electronic supplementary information (ESI) available. See DOI: 10.1039/c7ra02495a physical stimuli can also help to induce the formation of chiral assemblies. Such stimuli include the control of the interfacial tension of molecules at air-water interface with LangmuirBlodgett method, ${ }^{4}$ tuning the stirring directions of the solution and irradiating molecules with circular polarized light. ${ }^{5}$

Apart from the inherent and induced helical assemblies, there is also a special kind of helical assemblies formed by achiral $\pi$-conjugated molecules that their helical selfassemblies are formed by the asymmetric packing of achiral building blocks without incorporating chiral additives or external stimuli. ${ }^{6}$ The helical supramolecular assemblies of $\pi$ conjugated molecules are of great importance in the rational design of nanodevices and optical systems. ${ }^{7}$ Helical assemblies from non-chiral $\pi$-conjugated molecules represent an important aspect of chiral induction. Understanding the underlying principles of the assembly can help to broaden our knowledge about the formation of helical assembly from achiral molecules.

Hexaphenylsilole (HPS) has high electron acceptability, fast electron mobility and is an ideal material for electrontransporting, light emitting layers and field effect transistors. ${ }^{8}$ Compared with other kind of fluorescent molecules, HPS has significant AIE property and their fluorescence emission becomes several hundred time enhanced upon aggregation. ${ }^{9}$ This privilege has made this kind of molecule an ideal material for chemical sensors and electronic devices at film state. As revealed by the crystal structure of HPS, each silole core is decorated with six phenyl rings though single bonds. HPS molecule takes a propeller-like conformation due to the torsion between the peripheral phenyl rings and the silole plane. The peripheral phenyl rings at the ring carbons are all arranged either in a clockwise or anticlockwise manner. ${ }^{10}$ Thus HPS molecules 
are believed to have equal chance of both handedness and the chirality of HPS molecules is then cancelled by their opposite handedness in solution. Because of this view, the self-assembling behavior of HPS has been unnoticed ever since its synthesis and optical property were reported. Chiral HPS derivatives are then designed by deliberately introducing chiral attachment to the phenyl rings of silole backbone. ${ }^{11}$ Recently, we found that achiral $\pi$-conjugated molecule HPS can self-assemble into helical assemblies without incorporating chiral centers or adding chiral additives to the molecules. Upon aggregation, HPS selfassembles into helical fibers with hollow internalities which have fluorescence, CD and CPL properties. Theoretical modeling suggests that HPS molecules first aggregate into dimer blocks and different dimer blocks are further packed into helical assemblies. In the helical assemblies, inter-molecular van der Waals interaction plays an important role in the formation of dimer blocks and stable packing between neighboring blocks. In contrast to the dominant research on the AIE properties of HPS and its derivatives, ${ }^{12}$ their helical assemblies and CPL properties are unnoticed. The discovery of the helical assemblies formed by HPS will undoubtedly update and richen the already unique optical activities of this novel molecule (Scheme 1).

\section{Experimental section}

\section{Materials}

HPS and its derivative HPS-MR were synthesized by ring-closing reactions of 1,4-dilithio-2,3,4,5-tetraphenyl-1,3-butadiene with dichlorodiphenylsilane as reported previously. ${ }^{9}$ All the reagents were purchased from Aldrich and used without further purification.

\section{Instrumental analysis}

UV-vis spectra. A stock THF solution of $10^{-4} \mathrm{M}$ HPS and HPSMR was prepared for UV measurement, respectively. Film samples were prepared by casting $100 \mu \mathrm{L}$ THF solution of HPS

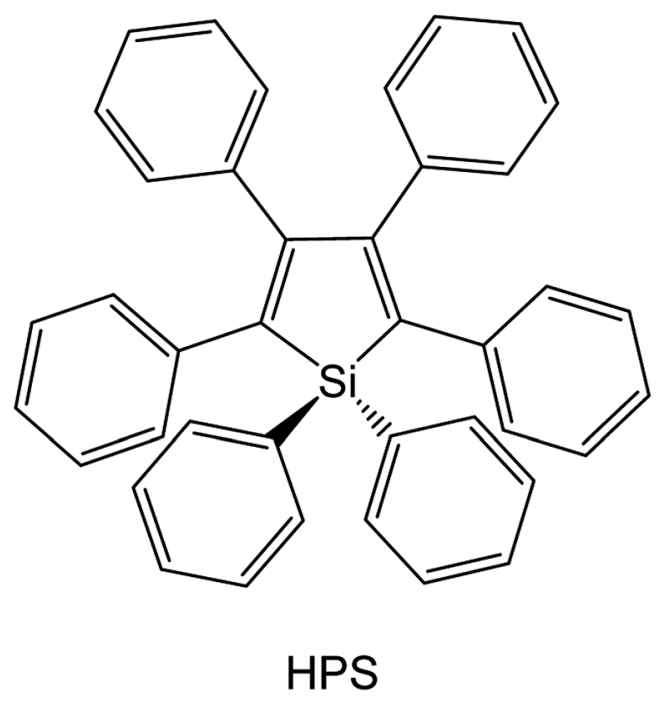

Scheme 1 Chemical structure of HPS. with the concentration of $10^{-3} \mathrm{M}$ to a quartz slide and allowed to evaporate under ambient condition. All the UV-vis measurements were carried out with a UV-2600 spectrometer (Shimadzu, Japan).

Circular dichroism (CD) measurement. Film samples for CD measurements were prepared by dropping $100 \mu \mathrm{L}$ THF solution of HPS and HPS-MR with the concentration of $10^{-4} \mathrm{M}$ on clean quartz slide, respectively. CD spectra were recorded on a Chirascan spectrometer (Applied Photophysics, England).

Circularly polarized luminescence (CPL) measurement. Film samples were prepared by casting $100 \mu \mathrm{L}$ THF solution of HPS with the concentration of $10^{-3} \mathrm{M}$ to a quartz slide and allowed to evaporate under ambient condition. CPL spectra of the films were recorded at $50 \mathrm{~nm} \mathrm{m^{-1 }}$ scan speed with a commercialized instrument JASCO CPL-200 at room temperature with the resolution of $15 \mathrm{~nm}$.

Atomic force microscope (AFM) measurement. AFM samples were prepared by dropping a droplet of $5 \mu \mathrm{L}$ THF solution of HPS with the concentration of $10^{-5} \mathrm{M}$ to the surface of newly cleaved mica under ambient conditions. After $5 \mathrm{~min}$ of incubation, the sample was dried with nitrogen gas. Images were collected with a Bruker Multimode-VIII AFM (Bruker, U. S. A.) operating in tapping mode or ScanAsyst mode.

Transmission electron microscope (TEM) measurement. A stock THF solution of $10^{-4}$ M HPS and HPS-MR was prepared. Aliquots of the stock solution were transferred to $10 \mathrm{~mL}$ volumetric asks. After appropriate amounts of THF were added, distilled water was added dropwise with vigorous stirring to yield $10^{-5} \mathrm{M}$ solutions with different water fractions. TEM samples were prepared by dropping a droplet of $5 \mu \mathrm{L}$ the above solution with different contents of water to the copper mesh coated with carbon films under ambient conditions. After evaporation naturally, the samples were examined by Tecnai $\mathrm{G}^{2}$ Spirit TEM at an accelerating voltage of $120 \mathrm{kV}$.

Theoretical modeling. The modeling of the assembly process contains two parts: molecular dynamics simulation of the conformations and identification of the packing manners of HPS molecules. The first part is performed with GROMACS package (version 4.6.5). The second part including the algorithm development and code implementation are all conducted by Luming Meng.

\section{Results and discussion}

\section{UV-vis and CD spectra}

The optical properties of HPS are studied with UV-vis and CD spectrometer in solution and at film state as well. As shown in Fig. 1, HPS exhibits two absorption peaks at $\sim 250 \mathrm{~nm}$ and $\sim 360 \mathrm{~nm}$ in solution and at film state corresponding to the phenyl groups connecting to silicon atom and silacyclopentadiene rings, respectively. The absorption of the film has a slight red shift compared with that in solution, suggesting that aggregation might be formed. HPS shows no CD signals in its THF solutions, but has obvious absorption of its cast film, as given in Fig. 1B. The cast film showed bisignate Cotton effect between the wavelength of $300 \mathrm{~nm}$ and $450 \mathrm{~nm}$, implying that helical conformation has been induced in the aggregation state 


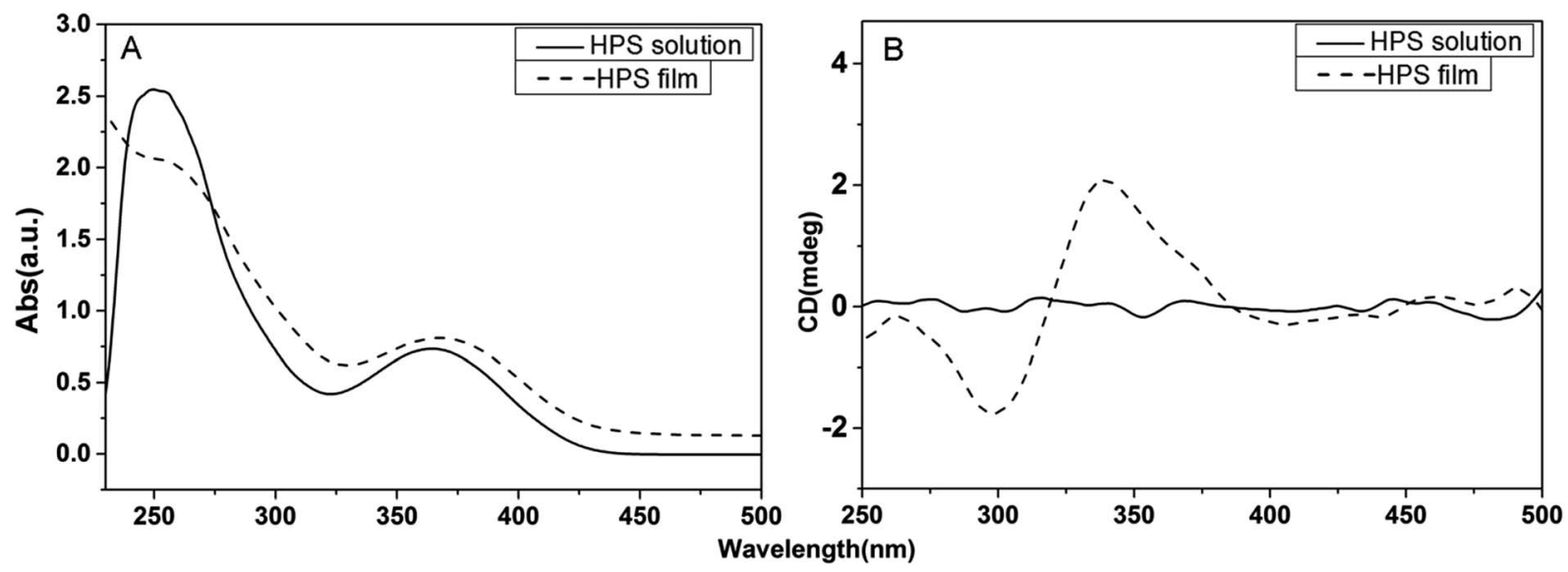

Fig. 1 The UV-vis absorption spectra (A) and CD spectra (B) of HPS in its THF solution (concentration: $10^{-4} \mathrm{M}$ ) and at cast film states.

of the molecules, suggesting that HPS has AICD property. ${ }^{13}$ Similar AICD effect was also found for the achiral HPS derivative bearing two methyl groups connected to silicone atom (HPSMR) at film state (in Fig. S1†), indicating that the AICD is typical for these kinds of achiral molecules at aggregation state.

As known from the crystal structures of HPS, the phenyl groups of each HPS molecule are arranged all in clockwise or anti-clockwise manner in the crystalline state. Molecules with these two manners of rotation will largely counterpart with the handedness of each other in solution, therefore giving no CD absorption. While in the cast film, the molecules are in aggregation state and their arrangements are not completely random. The molecules tend to have their phenyl rings show the same rotation direction in order for a maximum intermolecular interaction, namely the van der Waals interactions between neighboring phenyl rings. Thus it leads to a dominant clockwise or anti-clockwise arrangement, corresponding to the obvious CD absorption. The CD absorption of HPS is similar to that of the chiral HPS derivatives carrying chiral attachments, that is they only show AICD effect at film state. It suggests that the aggregation of HPS and its chiral derivatives are pivotal for the AICD effect.

\section{Circularly polarized luminescence}

Traditional luminescent molecules usually aggregate in a face to face manner and it is the main reason for aggregation caused quenching (ACQ) phenomenon. ${ }^{\mathbf{1 4}}$ While for HPS molecules, the neighboring phenyl rings are highly twisted in a propeller conformation due to their steric repulsion and the molecules are thus prevented from aggregation in a face to face manner, thus inhibiting the ACQ effect and generating AIE property. As the AIE property of HPS has already been well explored, here we mainly focus on other aggregation related optical property of the molecules. Generally molecules having both AICD absorption and luminescence may also have CPL property. CPL is the anisotropic emission of circularly polarized light from chiral molecular systems, and it provides specific information about the chirality of the luminogens of the molecules. ${ }^{15}$ The performance of CPL-active materials is generally evaluated by the emission dissymmetry factor $\left(g_{\mathrm{em}}\right)$, which is defined as $g_{\mathrm{em}}=2\left(I_{\mathrm{L}}\right.$ $\left.-I_{\mathrm{R}}\right) /\left(I_{\mathrm{L}}+I_{\mathrm{R}}\right)$, where $I_{\mathrm{L}}$ and $I_{\mathrm{R}}$ denotes the emission intensities of left- and right-handed circularly polarized light, respectively.

HPS possessing both AICD and AIE characteristics, is anticipated to possess CPL property. ${ }^{16}$ The CPL behavior of HPS is then studied both in solution and at the film state. As shown in Fig. 2, the solid film of HPS formed upon the evaporation of its THF solution gives negative CPL signal from 475 to $600 \mathrm{~nm}$, while the solution generated no obvious CPL signal. The emission dissymmetry factor of the film, $g_{\mathrm{em}}$, is in the range from 0.001 to -0.0125 and reaches the maximum -0.0125 at the wavelength of $550 \mathrm{~nm}$. While the HPS derivative HPS-MR is silent in CPL spectra both in solution and at film state (the data is not shown), suggesting that the phenyl groups connected to silicon are critical for the optical property of the molecule and when they are replaced by two methyl groups considerable influence on the CPL property is observed. To deliberately construct CPL bearing HPS-MR aggregates, chiral attachments need to be introduced to the AIE scaffold. ${ }^{11}$ Compared with reported CPL-active materials with chiral attachments, the $g_{\text {em }}$ of HPS is pretty high. Considering HPS does not need to suffer from ACQ effect, it is a promising CPL material.

\section{Helical assemblies}

CD spectra have revealed that HPS has AICD at film state. It is well-known that molecules with AICD usually forms helical aggregates. ${ }^{17}$ We then turned to AFM to explore the aggregates formed by HPS. We first studied the assembled structures formed by HPS upon the evaporation of its THF solution. Particles (not provided) are formed which are similar to what have been detected in acetone solution with nano particle analyzer previously. ${ }^{18}$ Helical fibers with left-handedness were also formed by HPS, as shown in Fig. 3A. The fibers were arranged in an extended way and thinner fibers further intertwine to form suprahelical fibers, which still retained lefthandedness. Because of the different levels of helical rotation, the helical fibers have a broad distribution of width and helical pitches. The formation of helical fibers corresponds well with the CD spectra, confirming that helical conformation has been 

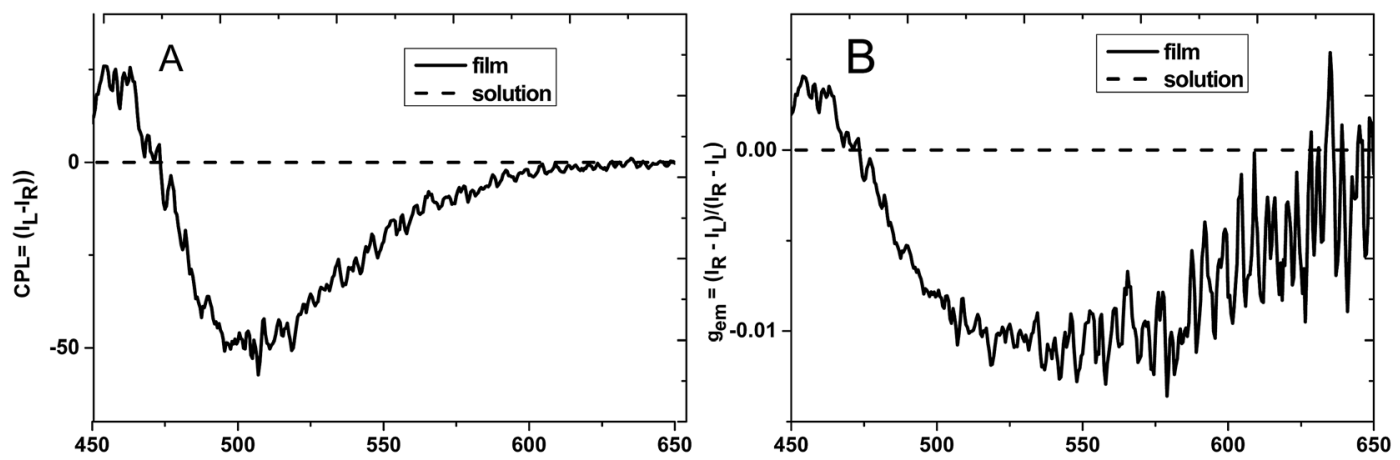

Wavelength $(\mathrm{nm})$

Fig. 2 Plots of (A) CPL and (B) CPL dissymmetry factor, $g_{\mathrm{em}}$, versus wavelength for HPS in THF solution with a concentration of $10^{-3} \mathrm{M}$ and at cast film.
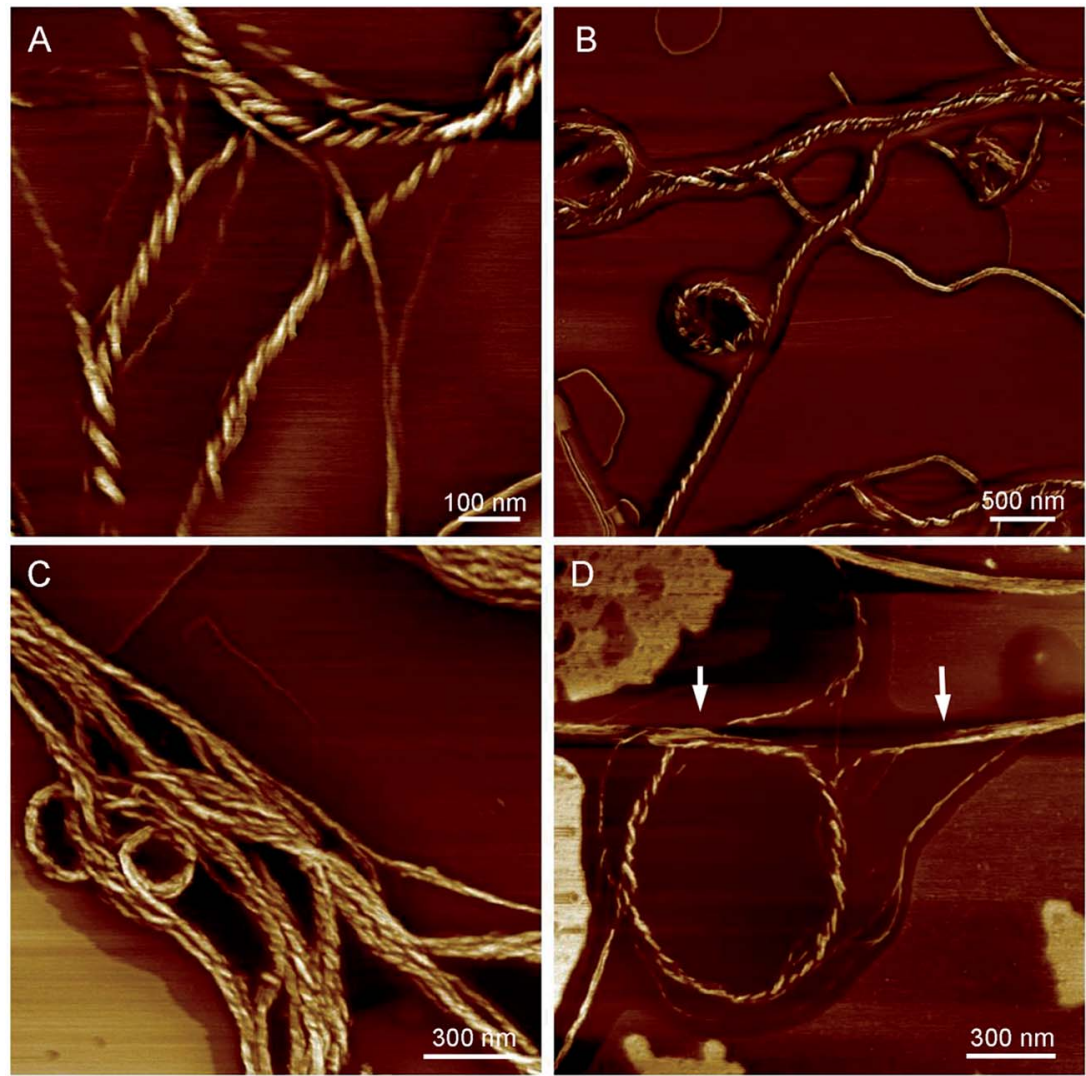

Fig. 3 AFM images of the self-assembled helical fibers formed by HPS on the evaporation of its different THF-water solution. The water content is $0 \%(A), 50 \%(B), 80 \%(C)$ and $90 \%$ (D), respectively, concentration: $10 \mu \mathrm{M}$, ribbons curled into fibers most significant at the area labeled with white arrows (D).

successfully amplified on the higher order architectures of HPS. The aggregation of HPS in the presence of different fractions of the poor solvent was also studied. As shown in Fig. 3B-D, helical assemblies are still formed upon the addition of poor solvent. Left-handed helical fibers can still be observed. Besides helical fibers, films were also formed which are jointed with the fibers. The combined ribbons which tend to curl into fibers are also found as shown with white arrows in Fig. 3D, suggesting that the helical fibers were formed by curling of the thin films.

In order to explore the internal structure and assembling behavior of the helical fibers of HPS, we employed TEM to study the morphology of these fibers. It can be seen clearly that HPS self-assembled into ribbon-like left-handedness helical nanofibers having the length of several micrometers, which also have different degree of distortion upon the evaporation of $\mathrm{THF} / \mathrm{H}_{2} \mathrm{O}$ 

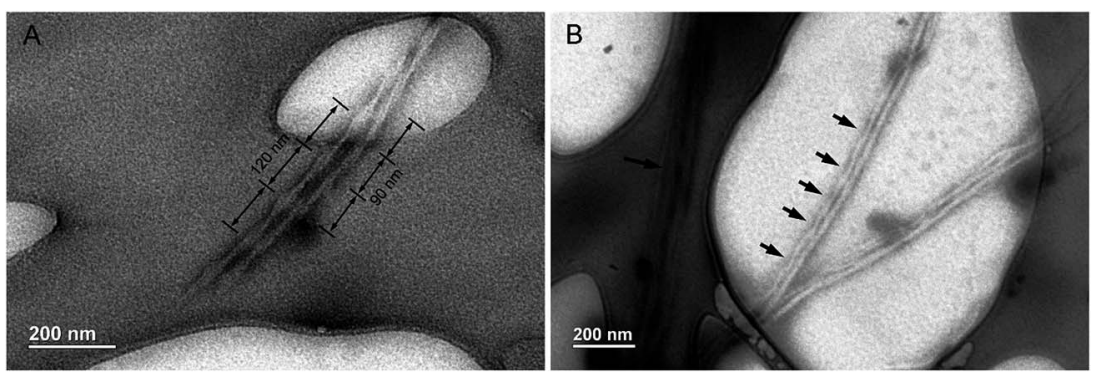

Fig. 4 TEM images of helical ribbons (A) and fibers of HPS (B) formed upon the evaporation of THF-water solution $\left(T H F / \mathrm{H}_{2} \mathrm{O}=1 / 4, \mathrm{v} / \mathrm{v}\right)$. Arrows indicate the helical pitches of the nanotubes.

$(1 / 4, v / v)$ mixture, as shown in Fig. 4A. Along with them, tubular morphology was also formed with the widths ranging from 20 to $50 \mathrm{~nm}$. The TEM images are consistent with AFM images and the hollow internalities of the fibers suggested that these helical fibers are formed by the curling of films.

\section{Modeling the packing of amorphous HPS aggregates}

We have studied the mechanism of aggregation process of HPS by AFM and TEM and revealed some important characteristics of the aggregates, such as the width and the period of the helical fiber. However, detailed process of the helical fibers still remains elusive due to the experimental limit. So we further perform computational calculation to model the architecture of aggregated fibers to provide more information about the underlying principle of the chiral assembly.

AFM and TEM results have provided significant clues for understanding the formation of the fiber structure. The geometric characteristics are mostly preserved among different segments of fibers from the experimental observation. This provides evidence of the existence of periodic architecture and repeated units in HPS aggregates. GROMACS package (version 4.6.5) was adopted to perform MD simulation to sample the conformations. ${ }^{19}$ Generally, the periodicity can be described by two types of components: translocation and rotation. In our modeled system, the orientation changes between neighboring units should be small, otherwise the size of fiber width will not reach up to as long as 50 nanometers (for example, if the rotation angle is $10^{\circ}$ and the size of each unit is $1 \mathrm{~nm}$ and the estimated diameter of fiber width will be smaller than $12 \mathrm{~nm}$ ). Thus one simple approximation is made that the periodicity existing in HPS aggregates, which can be mostly described by translocation. Finally, the total energy of the system should be optimized to maintain the stability of the aggregates.

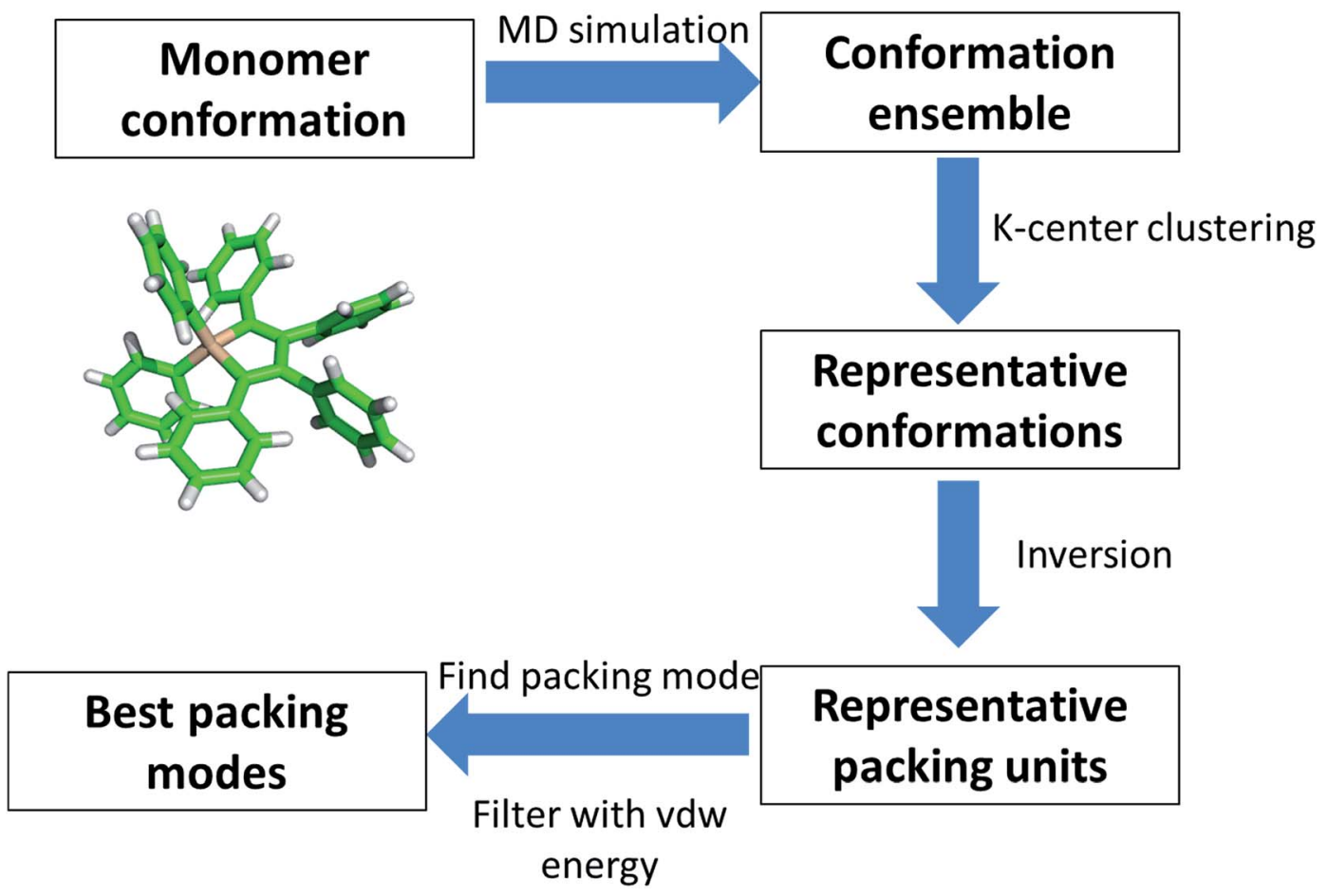

Fig. 5 Flow chart of modeling method. 

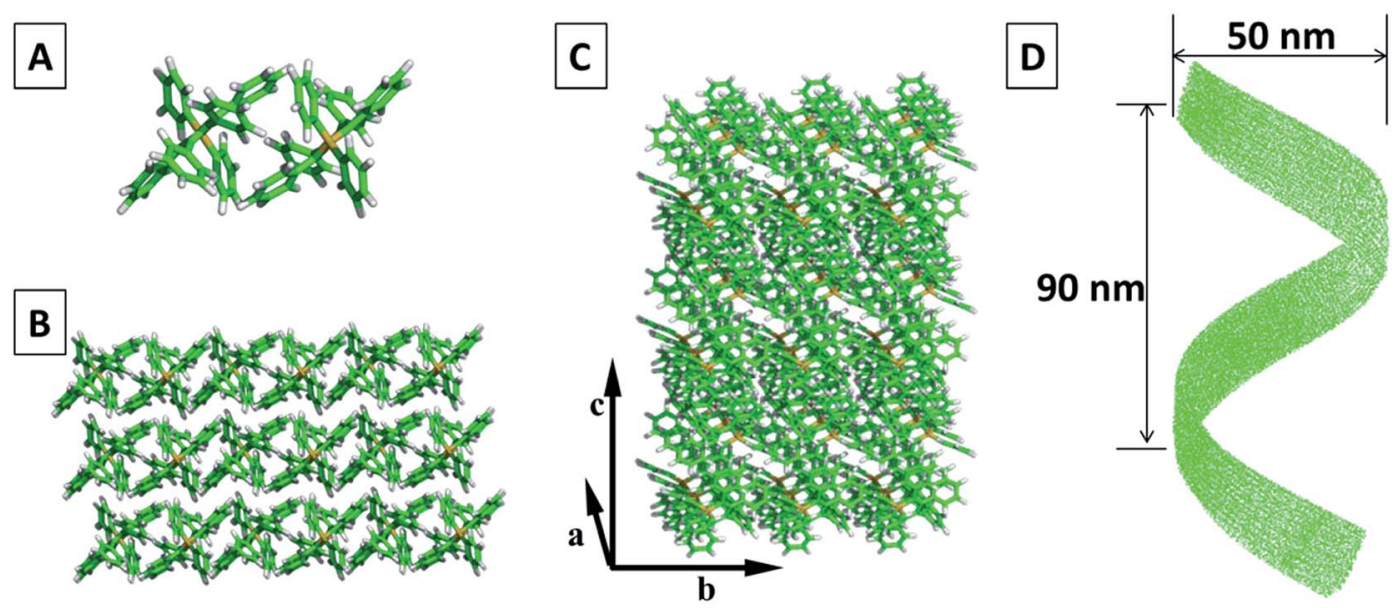

Fig. 6 Evolution of helical fibers from dimmers to molecular layers and helical fibers. The basic unit HPS aggregates with central symmetric property (A). Packing modes of molecular layers from different units from different views (B and C). Modeled helical fibers of HPS aggregates (D).

Combing these analysis together, we envisage that HPS fibers are consisted of identical units that are packed together mostly with translocation with low energy. Considering the inversion symmetry between two HPS molecules in one lattice cell in crystal structure, we take the same symmetric property in the modeling process. Finally, we propose a work flow for the modeling of HPS fibers, which is summarized into four steps, as shown in Fig. 5. These four steps are: (1) sampling the conformations of HPS molecules in solution with molecular dynamics (MD) simulations. (2) Selection of representative conformations of HPS molecule from trajectories generated by MD simulation. (3) Construction of packing units for aggregation with representative conformations. (4) Optimization of the best packing modes with different packing units by considering the total energy of system. Validation of this method is presented in Fig. S4. $\dagger$

With the proposed work flow, we identified thousands of packing modes with different HPS conformations and chose the one with the lowest energy as the final result (see Fig. S4 for details $\dagger$ ). In this result, two HPS molecules are anti-parallel placed to form a basic unit (Fig. 6A). Multiple units are further packed together to form a periodic pattern with specified lattice parameter $(a=9.9 \AA, b=11.3 \AA, c=15.2 \AA, \alpha=$ $90.3^{\circ}, \beta=84.6^{\circ}, \gamma=71.2^{\circ}$ ) for each unit. With more molecules joining the packing, molecular layers are formed (Fig. 6B and C). The central symmetric arrangement of the dimmers in each unit cell will lead to the tilting of the molecular layers. The tilting will be amplified with more molecules in the packing, which are then followed by the wrapping up of the layers on a much larger scale. Fully wrapping up of the layers (Fig. 6C) gives the morphology of helical tubes, while partially wrapping up of the layers lead to the morphology of helical fibers or ribbons.

Through the calculation, it is found that van der Waals interaction between the phenyl groups mainly contributes to the formation of the packing unit and packing mode.

\section{Conclusion}

We have provided the finding of the supramolecular chirality from achiral AIE molecule HPS. HPS was found to show exceptional AICD and CPL property in the aggregation state. Upon evaporation, it self-assembled into fluorescent helical nanofiber and nanotubes. Theoretical modeling suggests that van der Waals interaction between HPS is mainly responsible for the chiral assembly.

\section{Acknowledgements}

This work was partially supported by the National Natural Science Foundation of China (21574085), and The National Natural Science Foundation of Guangdong province (2016A030312002).

\section{References}

1 (a) M. Liu, L. Zhang and T. Wang, Chem. Rev., 2015, 115, 7304; (b) M. Kumar and S. J. George, Chem. Sci., 2014, 5, 3025 .

2 (a) S. Datta, S. K. Samanta and S. Bhattacharya, Chem.-Eur. J., 2013, 19, 11364; (b) K. Watanabe, H. Lida and K. Akagi, Adv. Mater., 2012, 24, 6451; (c) S. C. J. Meskers, E. Peeters, B. M. W. Langeweld-Voss and R. A. J. Janssen, Adv. Mater., 2000, 12, 589.

3 (a) F. Wang, M. A. J. Gillisen, P. J. M. Stals, A. R. A. Palmans and E. W. Meijer, Chem.-Eur. J., 2012, 18, 11761; (b) D. Yang, Y. Zhao, K. Lv, X. Wang, W. Zhang, L. Zhang and M. Liu, Soft Matter, 2012, 12, 1170; (c) B. Isare, M. Linares, L. Zargarian, S. Fermandjian, M. Miura, S. Motohashi, N. Vanthuyne, B. Lazzaronni and L. Bouteiller, Chem.-Eur. J., 2010, 16, 173; (d) P. J. M. Stals, J. C. Everts, R. D. Bruijin, I. A. W. Filot, M. M. J. Smulders, R. Martín-Rapún, E. A. Pidko, T. F. A. Greef, A. R. A. Palmans and E. W. Meijer, Chem.-Eur. J., 2010, 16, 810; (e) X. Xi, L. Jiang, W. Sun and Z. Shen, Chirality, 2007, 19, 521. 
4 X. Liu, T. Wang and M. Liu, Phys. Chem. Chem. Phys., 2011, 13, 16520.

5 Z. Chen, T. Wang, L. Shi, Z. Tang and M. Liu, Chem. Sci., 2015, 6, 4267.

6 (a) D. M. Bassani, J. Lehn, G. Baum and D. Fenske, Angew. Chem., Int. Ed. Engl., 1997, 36, 1845; (b) Q. Hu, Y. Wang, J. Jia, C. Wang, L. Feng, R. Dong, X. Sun and J. Hao, Soft Matter, 2012, 8, 11492; (c) W. Zhao, L. Feng, L. Xu, W. Xu, X. Sun and J. Hao, Langmuir, 2015, 31, 5748; (d) T. Shiraki, A. Dawn, Y. Tsuchiya, T. Yamanoto and S. Shinkai, Chem. Commun., 2012, 48, 7091; (e) B. Song, B. Liu, Y. Jin, X. He, D. Tang, G. Wu and S. Yin, Nanoscale, 2015, 7, 930; $(f)$ T. Shiraki, Y. Tsuchiya, T. Noguchi, S. Tamaru, N. Suzuki, M. Taguchi, M. Fujiki and S. Shinkai, Chem.-Asian J., 2014, 9, 218; $(g)$ A. Duong, M. A. Kobaisi and S. V. Bhosale, Sci. Rep., 2015, 5, 15652; (h) Y. Zhao, N. A. Rahim, Y. Xia, M. Fujiki, B. Song, Z. Zhang, W. Zhang and X. Zhu, Macromolecules, 2016, 49, 3214; (i) Z. Shen, T. Wang and M. Liu, Angew. Chem., Int. Ed., 2014, 53, 13424; (j) Z. Shen, Y. Jiang, T. Wang and M. Liu, J. Am. Chem. Soc., 2015, 137, 16109.

7 (a) G. Liu, L. Zhu, W. J. C. Feng and Z. Wei, Angew. Chem., Int. Ed., 2016, 55, 2411; (b) K. Akagi and T. Mori, Chem. Rec., 2008, 8, 395.

8 (a) A. P. Sadimenko, Adv. Heterocycl. Chem., 2001, 79, 115; (b) V. Y. Lee, A. M. Ichionohe and N. Fukaya, J. Organomet. Chem., 2000, 611, 228; (c) C. Corriu, R. J. P. Reye and J. C. Young, Chem. Rev., 1993, 93, 1371.

9 J. Luo, Z. Xie, J. W. Y. Lam, L. Chen, H. Chen, C. Qiu, H. S. Kwok, Y. Liu, D. Zhu and B. Z. Tang, Chem. Commun., 2001, 1740.

10 J. Chen, C. C. W. Law, J. W. Y. Lam, Y. Dong, S. M. F. Lo, I. D. Williams, D. Zhu and B. Z. Tang, Chem. Mater., 2003, 15, 1535.

11 (a) J. Liu, H. Su, L. Meng, Y. Zhao, C. Deng, J. C. Y. Ng, P. Lu, M. Faisal, J. W. Y. Lam, X. Huang, H. Wu, K. S. Wong and
B. Z. Tang, Chem. Sci., 2012, 3, 2737; (b) J. C. Y. Ng, H. Li, Q. Yuan, J. Liu, C. Liu, X. Fan, B. S. Li and B. Z. Tang, J. Mater. Chem. C, 2014, 2, 4615; (c) H. Li, S. Xue, H. Su, B. Shen, Z. Cheng, J. W. Y. Lam, K. S. Wong, H. Wu, B. S. Li and B. Z. Tang, Small, 2016, 12, 6593.

12 (a) B. Z. Tang, X. Zhang, G. Yu, P. P. S. Lee, Y. Liu and D. Zhu, J. Mater. Chem., 2001, 11, 2874; (b) H. T. Nguyen and M. T. Nguyen, J. Phys. Chem. C, 2016, 120, 19792; (c) B. Chen, G. X. Feng, B. R He, C. Goh, S. D. Xu, G. RamosOrtiz, L. Aparicio-Ixta, J. Zhou, L. G. Ng, Z. J. Zhao, B. Liu and B. Z. Tang, Small, 2016, 12, 782; (d) J. Chen and Y. Cao, Macromol. Rapid Commun., 2007, 28, 1714.

13 (a) H. Li, J. Cheng, Y. Zhao, J. W. Y. Lam, K. S. Wong, H. Wu, B. S. Li and B. Z. Tang, Mater. Horiz., 2014, 1, 518; (b) H. Li, J. Cheng, H. Deng, E. Zhao, B. Shen, J. W. Y. Lam, K. S. Wong, H. Wu, B. S. Li and B. Z. Tang, J. Mater. Chem. C, 2015, 3, 2399; (c) B. S. Li, R. Wen, S. Xue, L. Shi, Z. Tang, Z. Wang and B. Z. Tang, Mater. Chem. Front., 2017, 1, 576.

14 J. B. Birks, Photophysics of Aromatic Molecules, Wiley, New York, 1970.

15 (a) H. Maeda, Y. Bando, K. Shimomura, I. Yamada, M. Naito, K. Nobusawa, H. Tsumatori and T. Kawai, J. Am. Chem. Soc., 2011, 133, 9266; (b) T. Ikeda, T. Masuda, T. Hirao, J. Yuasa, H. Tsumatori, T. Kawai and T. Haino, Chem. Commun., 2012, 6025; (c) X. Liu, J. Jiao, X. Jiang, J. Li, Y. Cheng and C. Zhu, J. Mater. Chem. C, 2013, 1, 4713.

16 J. Roose, B. Z. Tang and K. S. Wong, Small, 2016, $12,6495$. 17 (a) Y. Zhang, S. Li, M. Ma, M. Yang, Y. Wang, A. Hao and P. Xing, New J. Chem., 2016, 40, 5568; (b) J. Ryu, L. Tang, E. Lee, H. Kim and M. Lee, Chem.-Eur. J., 2008, 14, 871.

18 J. Liu, J. W. Y. Lam and B. Z. Tang, J. Inorg. Organomet. Polym., 2009, 19, 249.

19 B. Hess, C. Kutzner, D. van der Spoel and E. Lindahl, J. Chem. Theory Comput., 2008, 4, 435. 